\section{\begin{tabular}{l|l|l} 
Received: & Revised: & Accepted:
\end{tabular}}

22 April 2020 | 27 July 2020 | 18 August 2020
2020 The Authors. Published by the British Institute of Radiology under the terms of the Creative Commons Attribution-NonCommercial 4.0 Unported License http://creativecommons.org/licenses/by-nc/4.O/, which permits unrestricted non-commercial reuse, provided the original author and source are credited.

FULL PAPER

\title{
Improving the image quality of DWI in breast cancer: comparison of multi-shot DWI using multiplexed sensitivity encoding to conventional single-shot echo- planar imaging DWI
}

\author{
${ }^{1}$ GABRIELLE C BAXTER, MPhys, ${ }^{2}$ ANDREW J PATTERSON, PhD, ${ }^{1}$ RAMONA WOITEK, MD, PhD, ${ }^{1}$ IRIS ALLAJBEU, MD, PhD, \\ ${ }^{2}$ MARTIN J GRAVES, PhD and ${ }^{1}$ FIONA GILBERT, MD, FRCR, FRCP
}

'Department of Radiology, University of Cambridge, Cambridge, United Kingdom

${ }^{2}$ Department of Radiology, Addenbrooke's Hospital, Cambridge, United Kingdom

Address correspondence to: Fiona Gilbert

E-mail:fjg28@medschl.cam.ac.uk

\begin{abstract}
Objective: To compare diffusion-weighted images (DWI) acquired using single-shot echo-planar imaging (ss-EPI) and multiplexed sensitivity encoding (MUSE) in breast cancer.

Methods 20 females with pathologically confirmed breast cancer (age $51 \pm 12$ years) were imaged with ss-EPI-DWI and MUSE-DWI. ADC, normalised ADC (nADC), blur and distortion metrics and qualitative image quality scores were compared. The Crété-Roffet and Mattes mutual information metrics were used to evaluate blurring and distortion, respectively. In a breast phantom, six permutations of MUSE-DWI with varying parallel acceleration factor and number of shots were compared. Differences in $A D C$ and $n A D C$ were compared using the coefficient of variation in the phantom and a paired t-test in patients. Differences in blur, distortion and qualitative metrics were analysed using a Wilcoxon signedrank test.
\end{abstract}

Results: There was a low coefficient of variation $(<2 \%)$ in ADC between ss-EPI-DWI and all MUSE-DWI permutations acquired using the phantom. 22 malignant and three benign lesions were identified in 20 patients. ADC values measured using MUSE were significantly lower compared to ss-EPI for malignant but not benign lesions $(p<0.001, p=0.21)$. nADC values were not significantly different ( $p=0.62, p=0.28$ ). Blurring and distortion improved with number of shots and acceleration factor, and significantly improved with MUSE in patients ( $p<$ 0.001, $p=0.002$ ). Qualitatively, image quality improved using MUSE.

Conclusion: MUSE improves the image quality of breast DWI compared to ss-EPI.

Advances in knowledge: MUSE-DWI has superior image quality and reduced blurring and distortion compared to ss-EPI-DWI in breast cancer.

\section{INTRODUCTION}

Diffusion-weighted imaging (DWI) is a promising non-contrast MRI technique in the detection of breast cancer. ${ }^{1,2}$ However, DWIs acquired using single-shot echo-planar imaging (ss-EPI) are limited by image artefacts and suffer from blurring due to $\mathrm{T}_{2}{ }^{*}$ decay during readout. The relatively low spatial resolution results in the averaging of tumour volumes with nearby breast tissues, particularly for small lesions and non-mass enhancements which are prone to volume-averaging affects due to the large voxel sizes of DWI compared to dynamic contrast-enhanced MRI (DCE-MRI). ${ }^{3}$ In order for diffusion MRI to be clinically relevant, it must be able to detect and characterise all lesions, even small lesions.

Parallel imaging techniques (such as $\mathrm{SENSE}^{4}$ ) aim to reduce artefacts in images acquired using EPI by reducing the length of the echo train and therefore reducing the $\mathrm{T}_{2}{ }^{*}$ blurring. However, the extent to which parallel imaging can improve image quality is limited by the coil hardware. Multi-shot techniques also attempt to reduce the length of the echo train by acquiring $k$-space in a number of segments. These techniques must account for motion between shots, often using a 'navigator' pulse to acquire 
Figure 1. Axial MUSE-DWI (two shots, acceleration factor 1.5) images of the A) $T_{1}$ relaxation and B) diffusion phantom inserts. The dotted line refers to the coronal slice C) with contents of the 16 vials labelled. Percentages refer to the percentage of polyvinylpyrrolidone (PVP) in water.
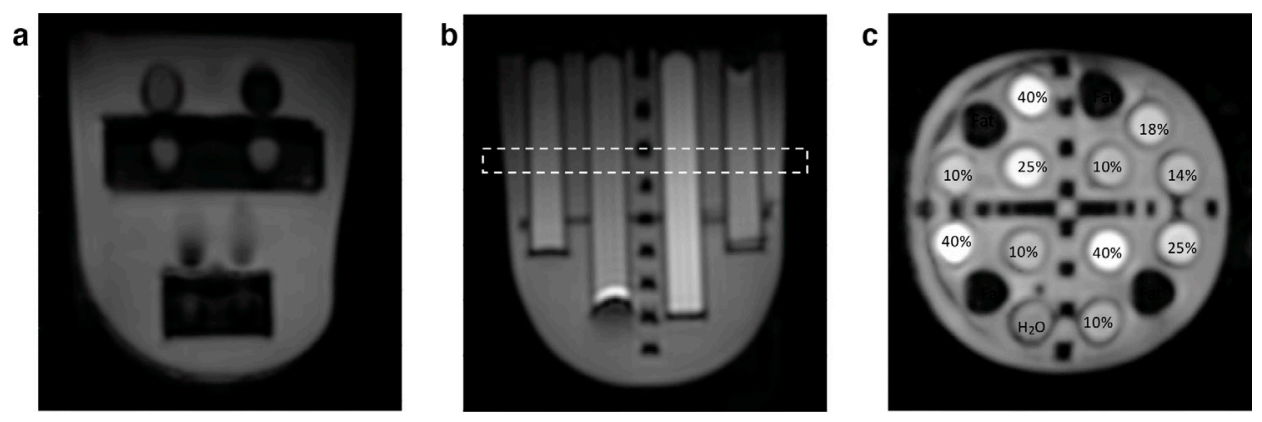

a low-resolution image that can be used to correct for motioninduced phase errors between shots.

Multiplexed sensitivity encoding (MUSE), a multi-shot segmented EPI technique, expands on existing sensitivityencoding techniques by acquiring $k$-space with an interleaved trajectory with the aim of achieving better spatial resolution and high signal-to-noise ratio (SNR) without the need for navigator pulses between interleaves. ${ }^{5}$ Benefits of a navigator-free method include an increase in efficiency of acquisition as navigators require $30-40 \mathrm{~ms}$ per excitation. ${ }^{6}$ Similar to parallel acceleration, MUSE is limited by the design of the receiver coil and the number of coil components. While MUSE has not yet been investigated in the breast, a number of studies have investigated the improvement in image quality and diagnostic performance of RESOLVE, a readout-segmented EPI technique. ${ }^{3,7-10}$

This study aimed to compare the image quality of DWI acquired using ss-EPI and MUSE, and investigate the effect of MUSE, used in conjunction with parallel acceleration, on the quantification of the apparent diffusion coefficient (ADC) in a breast phantom and in malignant, benign and normal breast tissue in a small cohort of patients. In addition, an optimised parallel acceleration factor and number of shots for MUSE-DWI were determined.

\section{METHODS AND MATERIALS}

\section{Phantom study}

Phantom study design

The effect of varying parallel acceleration factor and number of shots was first evaluated in a phantom. The phantom used in this study was manufactured by QMD (High Precision Devices, Inc., Boulder, CO), and consisted of two silicon breast inserts designed to investigate diffusion, distortion and $\mathrm{T}_{1}$ relaxation. The diffusion insert contained 16 vials of solutions arranged in two concentric rings with diameter and length $15 \times 110 \mathrm{~mm}$ (inner ring) and $15 \times 80 \mathrm{~mm}$ (outer ring) (Figure 1). 12 vials contained varying concentrations of polyvinylpyrrolidone (PVP) in water $(0,10,14,18,25$, and $40 \%)$ to mimic the apparent diffusion coefficients of benign and malignant tumours found in the literature and four vials contained a fat mimic. The interstitial space was filled with a solution of $35 \%$ corn syrup in water to mimic fibroglandular tissue. The phantom was kept in the scanner room at room temperature $\left(18^{\circ} \mathrm{C}\right)$.

Table 1. Scan parameters

\begin{tabular}{|c|c|c|c|c|c|}
\hline & \multicolumn{2}{|c|}{ Phantom studies } & \multicolumn{3}{|c|}{ Patient studies } \\
\hline & ss-EPI-DWI & MUSE-DWI & ss-EPI-DWI & MUSE-DWI & DCE-MRI \\
\hline Slice Orientation & Axial & Axial & Axial & Axial & Axial \\
\hline b-values $\left(\mathrm{s} / \mathrm{mm}^{2}\right)$ & 0,800 & 0,800 & 0,800 & 0,800 & - \\
\hline TR/TE (ms) & $4000 / 51$ & $4000 / 54-55$ & $3714-14853 / 60.7$ & $8203-16715 / 71.3$ & $5.1 / 2.4$ \\
\hline Matrix size & $160 \times 160$ & $160 \times 160$ & $96 \times 128$ & $160 \times 160$ & $384 \times 384$ \\
\hline Field-of-view $\left(\mathrm{mm}^{2}\right)$ & $360 \times 360$ & $360 \times 360$ & $360 \times 360$ & $360 \times 360$ & $360 \times 360$ \\
\hline Slice thickness $(\mathrm{mm})$ & 3.5 & 3.5 & 3.5 & 3.5 & 2.0 \\
\hline Acceleration factor & 2 & $1,1.5,2$ & 2 & 1.5 & 2.5 \\
\hline Number of shots & 1 & 2,3 & 1 & 2 & \\
\hline Acquisition Time & $1 \mathrm{~min} 8 \mathrm{~s}$ & $1 \min 52 \mathrm{~s}, 2 \min 48 \mathrm{~s}$ & $6-8 \min$ & $8 \mathrm{~min}$ & $9 \min$ \\
\hline
\end{tabular}

SS-EPI: single-shot echo-planar imaging, DWI: diffusion-weighted imaging, MUSE: multiplexed sensitivity encoding, DCE: dynamic contrastenhanced, TR: repetition time, TE: echo time. 
Figure 2. Axial images of the breast phantom using singleshot echo-planar imaging (ss-EPI), two or three shots and acceleration factors of 1, 1.5 and 2 . Insertion of the phantom in the breast coil causes the left phantom insert to bend slightly, this distortion is not caused by imaging technique.
2 shots
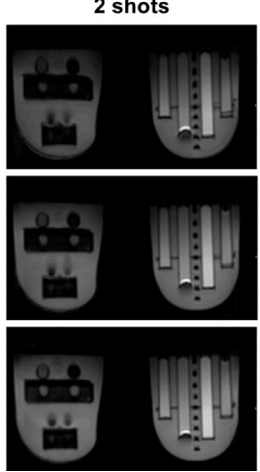

3 shots

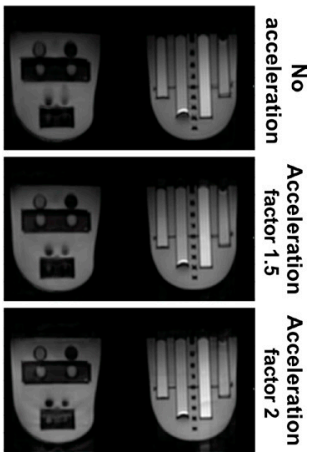

All phantom acquisitions were performed on a 3T system (Discovery MR 750, GE Healthcare, Waukesha, WI, USA) using a dedicated bilateral eight-channel phased-array breast coil. DWI was performed using ss-EPI and six permutations of MUSE-DWI with varying parallel acceleration factors and numbers of shots (Table 1$). \mathrm{A} \mathrm{T}_{1} \mathrm{~W}$ image $(\mathrm{TR} / \mathrm{TE}=659 / 15.3$ $\mathrm{ms}$, matrix $=512 \times 512$, slice thickness $=3.5 \mathrm{~mm}$ ) was acquired using a fast spin echo (FSE) sequence as a geometric reference.

A measurement of blurring was obtained using the Crété-Roffet blur metric, ${ }^{11}$ previously adapted from the field of computer vision, ${ }^{12}$ for a central slice through the phantom for all acquisitions. The metric is quantified by comparing the perceptible levels of blurring between the input image and a version of the image which has been further blurred using a low-pass filter. Values of the blur metric range from 0 (sharp) to 1 (blurry). A measurement of distortion was obtained by calculating the Mattes Mutual Information (MI) metric ${ }^{13}$ between the $b=800 \mathrm{~s} /$ $\mathrm{mm}^{2}$ and $\mathrm{T}_{1} \mathrm{~W}$ images, resampled to the same matrix size as the DWI images. Values of the MI metric range from 0 (distorted) to 1 (not distorted). Metrics were calculated for each slice and averaged over the whole image volume.
For each of the 16 vials, regions of interest (ROIs) were drawn on axial images for each of the diffusion gels using Osirix (v.8.0.1, Pixmeo, Switzerland). Each ROI consisted of three rectangles drawn on contiguous slices, avoiding the edges of the vials to minimise partial volume effects. Generation of ADC maps and diffusion analyses were carried out using in-house software developed in MATLAB (The Mathworks, version 2019a). The mean ADC was measured for each ROI and the coefficient of variation calculated between all acquisitions.

\section{Patient study design}

Between October 2018 and October 2019, 20 females were scanned under an existing research ethics protocol. The local institutional review boards and ethics committees approved this study and written informed consent was obtained from all patients. Patients were recruited if they had pathologically confirmed invasive breast cancer and had no previous treatment (such as breast surgery or neoadjuvant chemotherapy).

All clinical acquisitions were performed on a $1.5 \mathrm{~T}$ system (MR 450W, GE Healthcare, Waukesha, WI) using an 8-channel breast coil. ss-EPI-DWI and MUSE-DWI were performed. Highresolution $\mathrm{T}_{1} \mathrm{~W}$ dynamic contrast-enhanced (DCE) images were also acquired. Scan parameters are given in Table 1.

\section{Image analysis}

\section{Qualitative analysis}

Two breast radiologists, each of whom had at least 5 years of experience, independently reviewed all the images. Both ss-EPI and MUSE images were scored based on three qualitative image criteria: lesion conspicuity, contrast between lesion and tissue and diagnostic confidence. All criteria were scored from 1 (poor) to 5 (excellent). Lesions were also assessed for multi-focality and multi-centricity.

\section{Quantitative analysis}

The Crété-Roffet blur metric was calculated for ss-EPI and MUSE images, and the MI distortion metric was calculated between ss-EPI and MUSE images and the corresponding DCE images, resampled to the same matrix size as the DWI images. Metrics

Table 2. Apparent diffusion coefficient values measured for each acquisition for varying PVP concentrations

\begin{tabular}{|c|c|c|c|c|c|c|c|c|}
\hline \multirow{4}{*}{$\begin{array}{l}\text { PVP concentration } \\
(\%)\end{array}$} & \multicolumn{7}{|c|}{ Apparent Diffusion Coefficient $\left(10^{-3} \mathrm{~mm}^{2} / \mathrm{s}\right)$} & \multirow{4}{*}{$\begin{array}{c}\text { Coefficient of variation } \\
(\%)\end{array}$} \\
\hline & \multirow[b]{3}{*}{ ss-EPI } & \multirow{2}{*}{\multicolumn{3}{|c|}{$\begin{array}{c}\text { MUSE two shots } \\
\text { Acceleration Factor }\end{array}$}} & \multirow{2}{*}{\multicolumn{3}{|c|}{$\begin{array}{l}\text { MUSE three shots } \\
\text { Acceleration Factor }\end{array}$}} & \\
\hline & & & & & & & & \\
\hline & & 1 & 1.5 & 2 & 1 & 1.5 & 2 & \\
\hline 0 & 2.40 & 2.39 & 2.43 & 2.41 & 2.42 & 2.43 & 2.41 & 1.2 \\
\hline 10 & 1.74 & 1.75 & 1.79 & 1.79 & 1.79 & 1.81 & 1.82 & 1.5 \\
\hline 14 & 1.58 & 1.48 & 1.48 & 1.48 & 1.49 & 1.50 & 1.51 & 1.9 \\
\hline 18 & 1.37 & 1.29 & 1.31 & 1.31 & 1.31 & 1.32 & 1.33 & 1.8 \\
\hline 25 & 1.19 & 1.09 & 1.10 & 1.11 & 1.11 & 1.12 & 1.13 & 1.6 \\
\hline 40 & 0.67 & 0.60 & 0.70 & 0.69 & 0.69 & 0.66 & 0.67 & 1.6 \\
\hline
\end{tabular}

PVP: polyvinylpyrrolidone, ss-EPI: single-shot echo-planar imaging, MUSE: multiplexed sensitivity encoding 
Figure 3. Values of A) the Crété-Roffet blur metric and B) the mutual information metric measured for each phantom acquisition. S1, S2 and S3 refer to the use of 1, 2 and 3 shots. ACC1, ACC1.5 and ACC2 refer to the use of acceleration factor 1, 1.5 and 2. Circles indicate the acceleration factor and number of shots used in the clinical protocol.
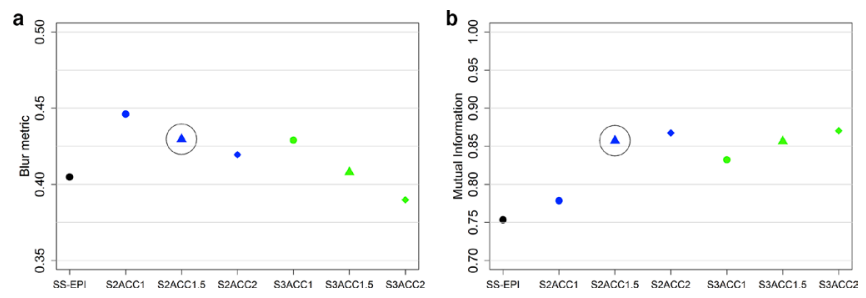

were calculated for each slice and averaged over the whole image volume.

ADC maps were generated using in-house software developed in MATLAB (v.2019a). ROIs were manually drawn on the ss-EPI and MUSE $b_{800}$ images by two breast radiologists in consensus with reference to DCE images to aid tumour delineation. As previously described by Wisner et $\mathrm{al}^{3}{ }^{3}$ to obtain a measurement of signal in normal tissue the ROI for each lesion was copied and placed on contralateral fibroglandular tissue. These ROIs were copied to the ADC maps and mean ADC values were measured for each lesion $\left(\mathrm{ADC}_{\text {lesion }}\right)$ and normal fibroglanduar tissue $\left(\mathrm{ADC}_{\text {tissue }}\right)$ ROI. To account for the difference in acquired voxel size, the normalised ADC (nADC) was calculated using $\mathrm{nADC}$ $=\mathrm{ADC}_{\text {lesion }} / \mathrm{ADC}_{\text {tissue }}$.

\section{Statistical analysis}

Qualitative and quantitative image quality metrics for images using ss-EPI or MUSE were compared on a per-patient basis. ROI sizes and ADC values were compared on a per-lesion basis. All comparisons used the paired t-test or a Wilcoxon signedrank test. The interobserver agreement of the qualitative scoring criteria was assessed using Cohen's $\kappa . p<0.05$ was considered statistically significant for all statistical tests. All statistical analyses were carried out using R (R v.3.1.3; R Foundation for Statistical Computing, Vienna, Austria).

\section{RESULTS}

Phantom results

Images acquired using ss-EPI and all permutations of MUSE are shown in Figure 2. ADC values measured using the phantom were consistent with values from the literature. ${ }^{14}$ The variation in ADC for each acquisition is shown in Table 2. There was a low coefficient of variation for measured ADC value $(<2 \%)$ for all PVP concentrations. The variation in MI metric and CrétéRoffet blur metric between acquisitions is shown in Figure 3. Both distortion and blurring improved (an increase in the MI metric and a decrease in the Crété-Roffet blur metric) with an increasing number of shots and acceleration factor. Increasing the acceleration factor when using three shots did not improve distortion. For clinical implementation, an acceleration factor of 1.5 and 2 shots were chosen as a pragmatic compromise between acquisition time and image quality.
Table 3. Patient and lesion characteristics

\begin{tabular}{|c|c|}
\hline Mean age in years \pm SD $($ range $)$ & $53.8 \pm 12.0(28-81)$ \\
\hline \multicolumn{2}{|l|}{ Total lesions $(n=24)$} \\
\hline Lesion size in $\mathrm{mm}($ mean $\pm \mathrm{SD})$ & $19.58 \pm 11.68$ \\
\hline \multirow[t]{2}{*}{ Range } & $10-56$ \\
\hline & $\mathrm{N}(\%)$ \\
\hline \multicolumn{2}{|l|}{ Malignant lesions $(n=22)$} \\
\hline Invasive ductal carcinoma & $11(50.0)$ \\
\hline Invasive lobular carcinoma & $8(36.4)$ \\
\hline $\begin{array}{l}\text { Invasive carcinoma with mixed ductal and } \\
\text { lobular features }\end{array}$ & $1(4.5)$ \\
\hline $\begin{array}{l}\text { Invasive ductal carcinoma with mucinous } \\
\text { differentiation }\end{array}$ & $1(4.5)$ \\
\hline High grade ductal carcinoma in situ & $1(4.5)$ \\
\hline \multicolumn{2}{|l|}{ Benign Lesions $(n=3)$} \\
\hline Fibroadenoma & $1(33.3)$ \\
\hline $\begin{array}{l}\text { Fibrocystic change with columnar cell } \\
\text { changes }\end{array}$ & $1(33.3)$ \\
\hline $\begin{array}{l}\text { Columnar and fibrocystic changes with } \\
\text { benign calcification }\end{array}$ & $1(33.3)$ \\
\hline \multicolumn{2}{|l|}{ Invasive breast cancer grade } \\
\hline Grade 1 & $3(14.3)$ \\
\hline Grade 2 & $14(66.7)$ \\
\hline Grade 3 & $4(19.0)$ \\
\hline \multicolumn{2}{|l|}{ ER status } \\
\hline Positive & $20(95.2)$ \\
\hline Negative & $1(4.8)$ \\
\hline \multicolumn{2}{|l|}{ PR status } \\
\hline Positive & $20(95.2)$ \\
\hline Negative & $1(4.8)$ \\
\hline \multicolumn{2}{|l|}{ HER2 status } \\
\hline Positive & $1(4.8)$ \\
\hline Negative & $20(95.2)$ \\
\hline
\end{tabular}

SD: standard deviation, ER: estrogen receptor, PR: progesterone receptor, HER2: human epidermal growth factor receptor 2

\section{Patient results}

20 patients (median age 51 years, range 28-81 years) were imaged. A total of 22 malignant lesions and three benign lesions were identified (median size $16 \mathrm{~mm}$, range $10-56 \mathrm{~mm}$ ). There were five patients who had more than one lesion. Two patients had a second lesion located in the contralateral breast. Three patients had a second lesion located in the same breast. Details of the patient and lesion characteristics are given in Table 3. Comparisons of image quality for an invasive ductal carcinoma, an invasive lobular carcinoma, and a patient with an invasive carcinoma with mixed ductal and lobular growth patterns and columnar and fibrocystic changes with benign calcification are shown in Figure 4. 
Figure 4. Post-gadolinium (Gd) DCE $T_{1} W$ images, DWI using ss-EPI and DWI using MUSE for a 60-year-old female with invasive ductal carcinoma (top), a 63-year-old female with invasive lobular carcinoma (middle), and a 51-year-old female with an invasive carcinoma with mixed ductal and lobular growth patterns (right arrow) and columnar and fibrocystic changes with benign calcification (left arrow) (bottom).

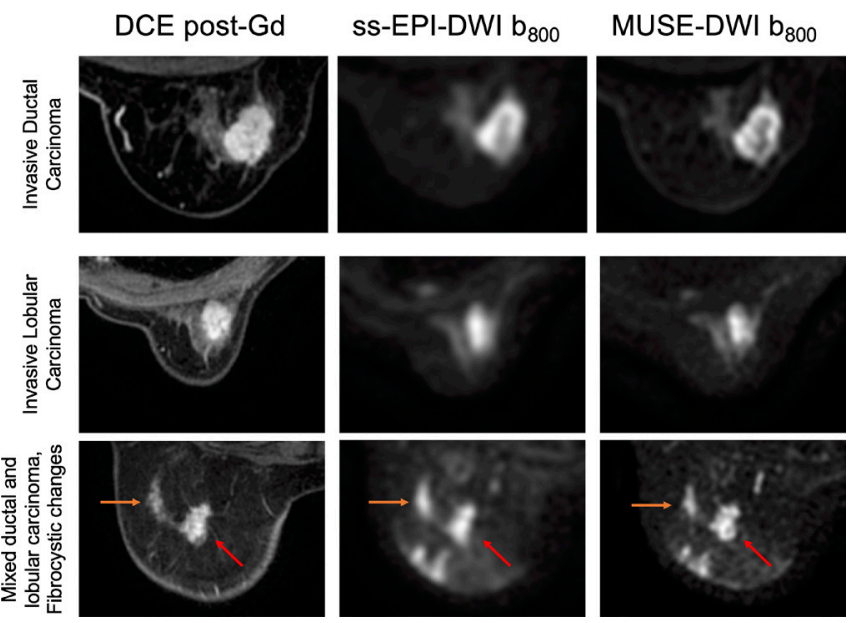

\section{Qualitative results}

Results of qualitative comparisons are given in Table 4. MUSE was superior to ss-EPI for contrast between lesion and tissue, and significantly superior for lesion conspicuity and diagnostic confidence criteria. Inter-reader agreement as measured by Cohen's $\kappa$ was higher for the scoring of MUSE images compared to ss-EPI.

\section{Quantitative results}

Results of quantitative comparisons are given in Table 5. The distributions of blur and distortion metrics using ss-EPI and MUSE are shown in Figure 5. The Crété-Roffet blur metric was significantly lower for MUSE-DWI than for ss-EPI-DWI ( $p$ $<0.001$ ), indicating less blurring. The MI metric was significantly higher for MUSE-DWI than for ss-EPI-DWI ( $p=0.002)$, indicating more similarity to DCE images and therefore less distortion.

\section{Comparison of ADC values: ss-EPI versus MUSE}

The distributions of ADC values measured using ss-EPI and MUSE are shown in Figure 6A. The mean ADC of malignant lesions was $1.49 \pm 0.30 \mathrm{~mm}^{2} / \mathrm{s}$ using ss-EPI and $1.23 \pm 0.27$ $\mathrm{mm}^{2} / \mathrm{s}$ using MUSE. The mean ADC of benign lesions was 1.73 $\pm 0.27 \mathrm{~mm}^{2} / \mathrm{s}$ using ss-EPI and $1.42 \pm 0.25 \mathrm{~mm}^{2} / \mathrm{s}$ using MUSE. $\mathrm{ADC}$ values measured using MUSE were significantly lower than those measured using ss-EPI for malignant lesions but not benign lesions ( $p<0.001$ and $p=0.21$, respectively). ADC values measured were in agreement with those in the literature, ${ }^{14}$ although the mean ADC for malignant lesions measured using ss-EPI was high.

The distributions of nADC values measured using ss-EPI and MUSE are shown in Figure 6B. The mean nADC of malignant lesions was $0.69 \pm 0.24$ using ss-EPI and $0.72 \pm 0.18$ using MUSE. The mean $\mathrm{nADC}$ of benign lesions was $0.79 \pm 0.21$ using ss-EPI and $0.97 \pm 0.23$ using MUSE. There was no significant difference in $\mathrm{nADC}$ values of malignant and benign lesions measured using ss-EPI or MUSE ( $p=0.62$ and $p=0.28$, respectively). The separation of the mean $\mathrm{nADC}$ values for malignant and benign lesions was greater using MUSE (0.25) than using ss-EPI (0.10).

\section{DISCUSSION}

In this study, we demonstrated through phantom and clinical experiments that the quality of DWI can be improved using MUSE by significantly reducing blurring and distortion. ADC values measured in the phantom and in patients were in agreement with those in the literature. ${ }^{14}$

Geometric distortion is prevalent in DWI due to the slow traversal through $k$-space along the phase-encoding direction when using ss-EPI. Accelerating the $k$-space trajectory through the use of a segmented-EPI technique such as MUSE can improve distortion compared to ss-EPI, as has been shown in previous studies implementing MUSE in the brain. ${ }^{15,16}$ Distortion has been evaluated in breast DWI by comparing lesion lengths in the anteroposterior and left-right directions ${ }^{7}$ or by using anatomic landmarks. ${ }^{10}$ However, a more robust technique using the MI metric, a similarity measure often used in the field of image registration, was used by Teruel et al when investigating the reduction of distortion after correction of $\mathrm{B}_{0}$ inhomogeneities. ${ }^{17}$ Distortion as measured by the MI metric in our study significantly improved using MUSE. In phantom studies, the best reduction in distortion was achieved using MUSE with three shots, however this was not clinically feasible due to the increase in acquisition time. A limitation of the MUSE technique is that the scan time increases proportional to the number of shots acquired. MUSE-DWI using simultaneous multi-slice excitation could be employed to reduce the acquisition time. This technique has been investigated in readout-segmented DWI in the breast. ${ }^{18}$

Blurring is also a limiting factor in the clinical use of ss-EPI DWI, affecting accurate measurement of the ADC. Low-resolution images result in ROIs that are averaged with adjacent normal fibroglandular tissue, leading to higher measured $\mathrm{ADC}$ values. While $\mathrm{ADC}$

Table 4. Qualitative comparisons

\begin{tabular}{|l|c|c|c|c|c|c|}
\hline Criteria & Score range & ss-EPI & MUSE & $p$-value & K ss-EPI & к MUSE \\
\hline Lesion conspicuity & $1-5$ & $3.5 \pm 1.1$ & $4.1 \pm 0.9$ & $<0.001$ & 0.05 & 0.4 \\
\hline Lesion-tissue contrast & $1-5$ & $3.8 \pm 1.2$ & $3.9 \pm 1.0$ & 0.86 & -0.007 & 0.3 \\
\hline Diagnostic confidence & $1-5$ & $3.7 \pm 1.1$ & $4.2 \pm 1.1$ & $<0.001$ & 0.08 & 0.2 \\
\hline
\end{tabular}

к: Cohen's א; MUSE: multiplexed sensitivity encoding,ss-EPI: single-shot echo-planar imaging.

$\mathrm{p}$-values were determined using a Wilcoxon signed-rank test. 
Figure 5. Comparison of A) Crété-Roffet blur metrics and B) Mattes Mutual Information distortion metrics using ss-EPI and MUSE.
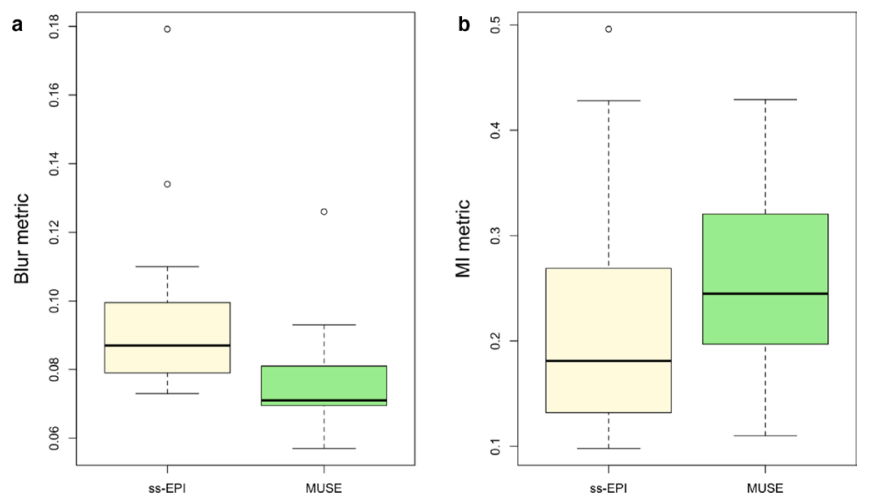

values measured in the breast phantom did not vary substantially using ss-EPI or MUSE when using the same set of ROIs, ADC values measured in patients were lower using MUSE, suggesting that the difference in ADC may be a result of improved resolution and therefore improved ROI delineation using MUSE. More accurate quantification of the ADC should improve the separation of $\mathrm{ADC}$ values between malignant, benign and normal fibroglandular tissue, as is seen by the greater separation of nADC values between malignant and benign breast lesions. Wisner et al similarly reported an improved separation of malignant and benign lesions using RESOLVE. ${ }^{3}$ In our study, there were not enough lesions to do an analysis of diagnostic performance. However, given that readoutsegmented techniques have been shown to have a better diagnostic performance compared to ss-EPI, ${ }^{8,9}$ we expect that MUSE will achieve a better diagnostic performance than ss-EPI.

Less blurring and higher spatial resolution will allow DWI to better detect smaller lesions and satellite lesions. Adhering to the EUSOBI guidelines, ${ }^{19}$ the patients included in this study were referred for MRI for preoperative local staging of ipsilateral and contralateral newly diagnosed breast cancers where patients had dense breasts or lobular cancers (resulting in a high number of lobular cancers in this study which does not reflect the natural prevalence of this type of cancer) as well as for problem-solving (equivocal findings
Figure 6. Comparison of the A) ADC and B) $\mathrm{AADC}$ values measured using ss-EPI versus MUSE for malignant and benign lesions and normal fibroglandular tissue.
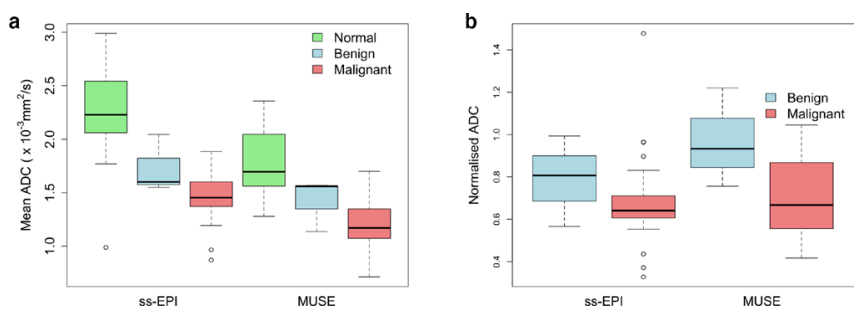

at mammography and ultrasound). In these cases, the improved image quality of MUSE-DWI can be used to better assess multifocality and multi-centricity, which impacts surgical planning. ${ }^{20}$ Reduced distortion may also allow for DWI to be used for morphological analysis, increasing the clinical utility of DWI.

There are limitations to this study. Firstly, low numbers of patients and lesions limit the conclusions that can be drawn. More benign lesions are required to evaluate diagnostic performance. Secondly, mostly mass type tumours were included in this study. Further work with a larger range of histopathological subtypes are required to prove the robustness of MUSE-DWI. Thirdly, we were not able to acquire our current clinical protocol with a matrix size of greater than $96 \times 128$ to match the matrix size used for MUSE as this resulted in a high level of distortion. This limits the conclusions that can be drawn from the improvement in blurring. However, phantom images acquired with ss-EPI and MUSE at the same resolution allowed for more direct comparisons of blurring and distortion. Finally, SNR and contrast-to-noise ratio (CNR) were not compared due to the varying parallel acceleration factors and SENSE reconstructions used among acquisitions, resulting in different noise characteristics.

\section{CONCLUSION}

In conclusion, the image quality of MUSE-DWI was superior to that of ss-EPI-DWI and geometric distortion and blurring were significantly reduced, although there was an increase in acquisition time. MUSE-DWI is a promising technique for the detection and

Table 5. Quantitative measures

\begin{tabular}{|l|c|c|c|}
\hline Parameter & ss-EPI & MUSE & $p$-value \\
\hline Mean ADC $\left(\times 10^{-3} \mathrm{~mm}^{2} / \mathrm{s}\right)$ & & & $<0.001$ \\
\hline Fibroglandular tissue & $2.24 \pm 0.45$ & $1.76 \pm 0.32$ & 0.21 \\
\hline Benign Lesions & $1.73 \pm 0.27$ & $1.42 \pm 0.25$ & $<0.001$ \\
\hline Malignant lesions & $1.49 \pm 0.30$ & $1.23 \pm 0.27$ & \\
\hline Mean normalised ADC & & & 0.28 \\
\hline Benign Lesions & $0.79 \pm 0.21$ & $0.97 \pm 0.23$ & 0.62 \\
\hline Malignant lesions & $0.69 \pm 0.24$ & $0.72 \pm 0.18$ & $<0.001$ \\
\hline Crété-Roffet blur metric & $0.10 \pm 0.02$ & $0.08 \pm 0.02$ & 0.002 \\
\hline Mattes Mutual Information metric & $0.19 \pm 0.12$ & $0.23 \pm 0.10$ & \\
\hline
\end{tabular}

ADC: apparent diffusion coefficient; MUSE: multiplexed sensitivity encoding,ss-EPI: single-shot echo-planar imaging.

$\mathrm{p}$-values were determined using a paired t-test. 
characterisation of breast lesions; however further work is required to evaluate the diagnostic performance of this technique using a larger range of histopathological subtypes of breast cancer.

\section{ACKNOWLEDGEMENTS}

We thank Gavin Houston and Simon Desonie of GE Healthcare for their support for this study. The authors would like to thank Amy Schiller and the team of breast MRI radiographers at Addenbrooke's and the Rosie hospital, Amy Frary, Johanna Field-Rayner, and the team of research nurses, and the patients for participating in this study. This work was supported by the National Institute for Health Research (Cambridge Biomedical Research Centre at the Cambridge University Hospitals NHS Foundation Trust). G.C.B. is funded by a GE Healthcare studentship. F.J.G. is sponsored by the National Institute for Health Research senior investigator award.

\section{FUNDING}

G.C.B. is funded by a GE Healthcare studentship.

\section{REFERENCES}

1. Partridge SC, Nissan N, Rahbar H, Kitsch AE, Sigmund EE. Diffusion-Weighted breast MRI: clinical applications and emerging techniques. J Magn Reson Imaging 2017; 45: 337-55. doi: https://doi.org/10.1002/jmri. 25479

2. Iima $\mathrm{M}$, Honda $\mathrm{M}$, Sigmund $\mathrm{EE}$, Ohno Kishimoto A, Kataoka M, Togashi K, et al. Diffusion MRI of the breast: current status and future directions. J Magn Reson Imaging 2020; 52: 70-90. doi: https://doi.org/ 10.1002/jmri.26908

3. Wisner DJ, Rogers N, Deshpande VS, Newitt DN, Laub GA, Porter DA, et al. HighResolution diffusion-weighted imaging for the separation of benign from malignant BI-RADS 4/5 lesions found on breast MRI at 3T. J Magn Reson Imaging 2014; 40: 674-81. doi: https://doi.org/10.1002/jmri.24416

4. Pruessmann KP, Weiger M, Scheidegger MB, Boesiger P. SENSE: sensitivity encoding for fast MRI. Magn Reson Med 1999; 42: 952-62. doi: https://doi.org/10.1002/( SICI)1522-2594(199911)42:5<952::AIDMRM16>3.0.CO;2-S

5. Chen N-K, Guidon A, Chang H-C, Song AW. A robust multi-shot scan strategy for highresolution diffusion weighted MRI enabled by multiplexed sensitivity-encoding (MUSE). Neuroimage 2013; 72: 41-7. doi: https://doi. org/10.1016/j.neuroimage.2013.01.038

6. Wu W, Miller KL. Image formation in diffusion MRI: a review of recent technical developments. J Magn Reson Imaging 2017; 46: 646-62. doi: https://doi.org/10.1002/jmri. 25664

7. Kim YJ, Kim SH, Kang BJ, Park CS, Kim HS, Son YH, et al. Readout-segmented echoplanar imaging in diffusion-weighted MR imaging in breast cancer: comparison with single-shot echo-planar imaging in image quality. Korean J Radiol 2014; 15: 403-10. doi: https://doi.org/10.3348/kjr.2014.15.4.403

8. Yamaguchi K, Nakazono T, Egashira R, Komori Y, Nakamura J, Noguchi T, et al.
Diagnostic performance of diffusion tensor imaging with Readout-segmented Echoplanar imaging for invasive breast cancer: correlation of ADC and FA with pathological prognostic markers. Magn Reson Med Sci 2017; 16: 245-52. doi: https://doi.org/10. 2463/mrms.mp.2016-0037

9. An YY, Kim SH, Kang BJ. Differentiation of malignant and benign breast lesions: added value of the qualitative analysis of breast lesions on diffusion-weighted imaging (DWI) using readout-segmented echoplanar imaging at 3.0 T. PLoS One 2017; 12: e0174681. doi: https://doi.org/10.1371/ journal.pone.0174681

10. Bogner W, Pinker-Domenig K, Bickel H, Chmelik M, Weber M, Helbich TH, et al. Readout-segmented echo-planar imaging improves the diagnostic performance of diffusion-weighted MR breast examinations at 3.0 T. Radiology 2012; 263: 64-76. doi: https://doi.org/10.1148/radiol.12111494

11. Crété-Roffet F, Dolmiere T, Ladret P. The Blur Effect: Perception and Estimation with a New No-Reference Perceptual Blur Metric The Blur Effect: Per-ception and Estimation with a New No-Reference Perceptual Blur Metric. SPIE Electronic Imaging Symposium Conf Human Vision and Electronic Imaging The Blur Effect: Perception and Estimation with a New No-Reference Perceptual Blur Metric. 2007. Available from: https://hal. archives-ouvertes.fr/hal-00232709 [21 October 2019].

12. McKay JA, Metzger GJ, Moeller S, et al. Adaptation of a computer vision blur metric to objectively compare high resolution DWI strategies in in vivo breast imaging. In: Proc. Intl. Soc. Mag. Reson. Med 2019; 27.

13. Mattes D, Haynor DR, Vesselle H, Lewellen TK, Eubank W. PET-CT image registration in the chest using free-form deformations. IEEE Trans Med Imaging 2003; 22: 120-8. doi: https://doi.org/10.1109/TMI.2003. 809072
14. Keenan KE, Wilmes LJ, Aliu SO, Newitt DC, Jones EF, Boss MA, et al. Design of a breast phantom for quantitative MRI. J Magn Reson Imaging 2016; 44: 610-9. doi: https://doi.org/ 10.1002/jmri.25214

15. Chang H-C, Sundman M, Petit L, Guhaniyogi S, Chu M-L, Petty C, et al. Human brain diffusion tensor imaging at submillimeter isotropic resolution on a 3Tesla clinical MRI scanner. Neuroimage 2015; 118: 667-75. doi: https://doi.org/10.1016/j.neuroimage.2015.06. 016

16. Chang H-C, Gaur P, Chou Y-hui, Chu M-L, Chen N-kuei. Interleaved EPI based fMRI improved by multiplexed sensitivity encoding (MUSE) and simultaneous multi-band imaging. PLoS One 2014; 9: e116378. doi: https://doi.org/10.1371/journal.pone.0116378

17. Teruel JR, Fjøsne HE, Østlie A, Holland D, Dale AM, Bathen TF, et al. Inhomogeneous static magnetic field-induced distortion correction applied to diffusion weighted MRI of the breast at 3T. Magn Reson Med 2015; 74: 1138-44. doi: https://doi.org/10.1002/mrm. 25489

18. Filli L, Ghafoor S, Kenkel D, Liu W, Weiland E, Andreisek G, et al. Simultaneous multi-slice readout-segmented echo planar imaging for accelerated diffusion-weighted imaging of the breast. Eur J Radiol 2016; 85: 274-8. doi: https://doi.org/10.1016/j.ejrad.2015.10.009

19. Mann RM, Balleyguier C, Baltzer PA, Bick U, Colin C, Cornford E, et al. Breast MRI: EUSOBI recommendations for women's information. Eur Radiol 2015; 25: 3669-78. doi: https://doi.org/10.1007/s00330-015-3807$\mathrm{z}$

20. Houssami N, Ciatto S, Macaskill P, Lord SJ, Warren RM, Dixon JM, et al. Accuracy and surgical impact of magnetic resonance imaging in breast cancer staging: systematic review and meta-analysis in detection of multifocal and multicentric cancer. JCO 2008; 26: 3248-58. doi: https://doi.org/10. 1200/JCO.2007.15.2108 